\title{
LXIX. On the integration and transformation of certain differential equations
}

\section{Rev. Brice Bronwin}

To cite this article: Rev. Brice Bronwin (1846) LXIX. On the integration and transformation of certain differential equations, Philosophical Magazine Series 3, 29:196, 494-500, DOI: $10.1080 / 14786444608645548$

To link to this article: http://dx.doi.org/10.1080/14786444608645548

册 Published online: 30 Apr 2009.

Submit your article to this journal $₫$

Џll Article views: 3

Q View related articles $₫$ 
to be distinguished from cumarine. On fusing these crystals with hydrate of potassa and testing the product of decomposition with sesquichloride of iron, the intense violet colour produced by the salicylic acid which had been formed showed with certainty the presence of cumarine in the original substance.

The hippuric acid found in the urine of graminivorous animals has in various ways been supposed to be derived from the benzoic acid imagined to exist in the Anthoxanthum odoratum. The transformation experienced by that acid in the animal conomy appeared in the highest degree to give support to this view.

It would be desirable to try if the same change takes place with cumarine. The narcotic action of this substance on the animal organism however prevented the making of any direct experiments.

LXIX. On the Integration and Transformation of certain Differential Equations. By the Rev. Brice Bronwin.*

THAVE lately discovered a very simple method of integraI ting and transforming certain differential equations, which I believe to be quite new. A similar method may be applied to the solution of equations in finite differences. Brief illustrations of it have been transmitted to the Mathematician and the Cambridge Mathematical Journal, but not yet published. The present paper contains an extension of the method as applied to differential equations. The following subsidiary formulæ will be required to facilitate the reductions. And it must be observed that $\mathrm{D}$ always stands for $\frac{d}{d x}$, and operates upon the whole of the quantity which follows it.

$$
\left.\begin{array}{l}
x \mathrm{D}^{n} y=\mathrm{D}^{n} x y-n \mathrm{D}^{n-1} y \\
x^{2} \mathrm{D}^{n} y=\mathrm{D}^{n} x^{2} y-2 n \mathrm{D}^{n-1} x y+n(n-1) \mathrm{D}^{n-2} y \\
x^{3} \mathrm{D}^{n} y=\mathrm{D}^{n} x^{3} y-3 n \mathrm{D}^{n-1} x^{2} y+3 n(n-1) \mathrm{D}^{n-2} x y-n \\
\quad(n-1)(n-2) \mathrm{D}^{n-3} y
\end{array}\right\} .
$$

These will be verified by actually performing the differentiations.

$$
\left.\begin{array}{l}
x \mathrm{D}^{-n} y=\mathrm{D}^{-n} x y+n \mathrm{D}^{-n-1} y \\
x^{2} \mathrm{D}^{-n} y=\mathrm{D}^{-n} x^{2} y+2 n \mathrm{D}^{-n-1} x y+n(n+1) \mathrm{D}^{-n-2} y
\end{array}\right\}
$$

\&c., which may be verified by successive differentiation.

We now proceed to give some examples.

* Communicated by the Author. 
Transformation of certain Differential Equations. $\quad 495$ Let

$$
x\left(\frac{d^{2} y}{d x^{2}}+k^{2} y\right)+2 p \frac{d y}{d x}=0,
$$

where $p$ is a positive integer. Make

Then, reducing by $(a$.$) , we have$

$$
y=\mathrm{D}^{2} u+k^{2} u=\left(\mathrm{D}^{2}+k^{2}\right) u \text {. }
$$

$$
\begin{aligned}
x \frac{d^{2} y}{d x^{2}}= & x \mathrm{D}^{4} u+k^{2} x \mathrm{D}^{2} u=\mathrm{D}^{4} x u-4 \mathrm{D}^{3} u+k^{2} \mathrm{D}^{2} x u-2 k^{2} \mathrm{D} u \\
& =\left(\mathrm{D}^{2}+k^{2}\right)\left(\mathrm{D}^{2} x u-4 \mathrm{D} u\right)+2 k^{2} \mathrm{D} u .
\end{aligned}
$$

In like manner

$$
2 p \frac{d y}{d x}=2 p \mathrm{D}^{3} u+2 p k^{2} \mathrm{D} u=\left(\mathrm{D}^{2}+k^{2}\right) 2 p \mathrm{D} u \text {. }
$$

Also

$$
\begin{aligned}
k^{2} x y & =k^{2} x \mathrm{D}^{2} u+k^{4} x u=k^{2} \mathrm{D}^{2} x u-2 k^{2} \mathrm{D} u+k^{4} x u \\
& =\left(\mathrm{D}^{2}+k^{2}\right) k^{2} x u-2 k^{2} \mathrm{D} u .
\end{aligned}
$$

Substituting these values in the given equation, it becomes

$$
\left(\mathrm{D}^{2}+k^{2}\right)\left\{\mathrm{D}^{2} x u+(2 p-4) \mathrm{D} u+k^{2} x u\right\}=0 \text {, }
$$

or

which is equivalent to

$$
\mathrm{D}^{2} x u+(2 p-4) \mathrm{D} u+k^{2} x u=0,
$$

$$
x\left(\frac{d^{2} u}{d \cdot x^{2}}+k^{2} u\right)+(2 p-2) \frac{d u}{d x}=0,
$$

an equation similar to the given one, $p$ being changed into $p-1$. Hence, by continuing to repeat the same operations, the last term may be taken away. If therefore $y=\left(\mathrm{D}^{2}+k^{2}\right)^{p} u$, we shall have $\frac{d^{2} u}{d x^{2}}+k^{2} u=0$, the integral of which is known. Again, let $\quad \frac{d^{2} y}{d x^{2}}+k x \frac{d y}{d x}-p k y=0$.

Here make $y=\mathrm{D} u+k x u=(\mathrm{D}+k x) u$. As before, reducing by $(a$ ), we have

$\frac{d^{2} y}{d x^{2}}=\mathrm{D}^{3} u+k \mathrm{D}^{2} x u=\mathrm{I}^{3} u+k x \mathrm{D}^{2} u+2 k \mathrm{D} u=(\mathrm{D}+k x) \mathrm{D}^{2} u$ $+2 k \mathrm{D} u$.

And

$$
\begin{aligned}
& k x \frac{d y}{d x}=k x \mathrm{D}^{2} u+k^{2} x \mathrm{D} x u=k \mathrm{D}^{2} x u-2 k \mathrm{D} u+k^{2} x \mathrm{D} x u \\
& =(\mathrm{D}+k x) k \mathrm{D} x u-2 k \mathrm{D} u .
\end{aligned}
$$

Substituting these values in the given equation, it becomes

$$
(\mathrm{D}+k x)\left(\mathrm{D}^{2} u+k \mathrm{D} x u-p k u\right)=0 \text {. }
$$

Or

$$
\mathrm{D}^{2} u+k \mathrm{D} x u-p k u=0,
$$

which is equivalent to 


$$
\frac{d^{2} u}{d x^{2}}+k x \frac{d u}{d x}-(p-1) k u=0 .
$$

This is similar to the proposed, $p$ being diminished of unity. By $p$ operations, therefore, the last term will be taken away. Hence if

then

$$
\begin{gathered}
y=(\mathrm{D}+k x)^{p} u, \\
\frac{d^{z} u}{d x^{2}}+k x \frac{d u}{d x}=0,
\end{gathered}
$$

an integrable equation.

To take a third example, let

Make

$$
x\left(\frac{d^{3} y}{d x^{3}}+k^{3} y\right)+3 p \frac{d^{2} y}{d x^{2}}=0 .
$$

We have

$$
\begin{aligned}
& \qquad \begin{aligned}
x \frac{d^{3} y}{d x^{3}} & =x \mathrm{D}^{6} u+k^{3} x \mathrm{D}^{3} u=\mathrm{D}^{6} x u-6 \mathrm{D}^{5} u+k^{3} \mathrm{D}^{3} x u-3 k^{3} \mathrm{D}^{2} u \\
& =
\end{aligned} \\
& \text { and } \left.\mathrm{D}^{3}+k^{3}\right)\left(\mathrm{D}^{3} x u-6 \mathrm{D}^{2} u\right)+3 k^{3} \mathrm{D}^{2} u \\
& \qquad \begin{aligned}
k^{3} x y & =k^{3} x \mathrm{D}^{3} u+k^{6} x u=k^{3} \mathrm{D}^{3} x u-3 k^{3} \mathrm{D}^{2} u+k^{6} x u \\
& =\left(\mathrm{D}^{3}+k^{3}\right) k^{3} x u-3 k^{3} \mathrm{D}^{2} u \text {. }
\end{aligned}
\end{aligned}
$$

With these values the given equation becomes

$$
\left(\mathrm{D}^{3}+k^{3}\right)\left\{\mathrm{D}^{3} x u+(3 p-6) \mathrm{D}^{2} u+k^{3} x u\right\}=0 ;
$$

or

$$
\mathrm{D}^{3} x u+(3 p-6) \mathrm{D}^{2} u+l^{3} x u=0 \text {; }
$$

or

$$
x\left(\frac{d^{3} u}{d x^{3}}+k^{3} u\right)+3(p-1) \frac{d^{2} u}{d x^{2}}=0 .
$$

Therefore if $y=\left(\mathrm{D}^{3}+k^{s}\right)_{k}^{p} u$, the proposed reduces to

$$
\frac{d^{3} u}{d x^{3}}+k^{3} u=0
$$

which we know how to integrate.

$$
\text { Next, let } \quad x \frac{d^{2} y}{d x^{2}}+(2 p+2 m \cdot x)\left(\frac{d y}{d x}+m y\right)=0 .
$$

Here we make $y=\left(\mathrm{D}^{2}+2 m \mathrm{D}+2 m^{2}\right) u$.

Then

$$
x \frac{d^{2} y}{d x^{2}}=x \mathrm{D}^{4} u+2 m x \mathrm{D}^{3} u+2 m^{2} x \mathrm{D}^{2} u
$$

$=\mathrm{D}^{4} x u-4 \mathrm{D}^{3} u+2 m \mathrm{D}^{3} x u-6 m \mathrm{D}^{2} u+2 m^{2} \mathrm{D}^{2} x u-4 m^{2} \mathrm{D} u$

$=\left(\mathrm{D}^{2}+2 m \mathrm{D}+2 m^{2}\right)\left(\mathrm{D}^{2} x u-4 \mathrm{D} u\right)+2 m \mathrm{D}^{2} u+4 m^{2} \mathrm{D} u$ $2 m x \frac{d y}{d x}=2 m x \mathrm{D}^{3} u+4 m^{2} x \mathrm{D}^{2} u+4 m^{3} x \mathrm{D} u$

$=2 m \mathrm{D}^{3} x u-6 m \mathrm{D}^{2} u+4 m^{2} \mathrm{D}^{2} x u-8 m^{2} \mathrm{D} u+4 m^{3} \mathrm{D} x u-4 m^{3} u$

$$
=\left(\mathrm{D}^{2}+2 m \mathrm{D}+2 m^{2}\right)\left(2 m \mathrm{D} x u-6 m^{u}\right)+4 m^{2} \mathrm{D} u+8 m^{3} u
$$




$$
\begin{aligned}
& 2 m^{2} x y=2 m^{2} x \mathrm{D}^{2} u+4 m^{3} x \mathrm{D} u+4 m^{4} x u \\
& =\left(\mathrm{D}^{2}+2 m \mathrm{D}+2 m^{2}\right) 2 m^{2} x u-4 m^{2} \mathrm{D} u-4 m^{3} u .
\end{aligned}
$$

Substituting these values in the given equation, and dividing the result by $\mathrm{D}^{2}+2 m \mathrm{D}+2 m^{2}$, a factor common to all the terms, we have

$\mathrm{D}^{2} x u+2 m \mathrm{D} x u+(2 p-4) \mathrm{D} u+2 m^{2} x u+(2 p-4) m u=0$;

or

$$
x \frac{d^{2} u}{d x^{2}}+(2 p-2+2 m x)\left(\frac{d u}{d x}+m u\right)=0,
$$

which is similar to the given equation, $p$ being changed into $p-1$. If therefore

$$
y=\left(\mathrm{D}^{2}+2 m \mathrm{D}+2 m^{2}\right)^{p} u,
$$

we shall have for the determination of $u$ the integrable equation

$$
\frac{d^{2} u}{d x^{2}}+2 m \frac{d u}{d x}+2 m^{2} u=0 .
$$

We will now apply the transformation $y=(\mathrm{D}+m) u$ to a few examples at once. This gives

$$
\begin{aligned}
x \frac{d^{2} y}{d x^{2}} & =x \mathrm{D}^{3} u+m x \mathrm{D}^{2} u=\mathrm{D}^{3} x u-3 \mathrm{D}^{2} u+m \mathrm{D}^{2} x u-2 m \mathrm{D} u \\
& =(\mathrm{D}+m)\left(\mathrm{D}^{2} x u-3 \mathrm{D} u\right)+m \mathrm{D} u \\
x \frac{d y}{d x} & =x \mathrm{D}^{2} u+m x \mathrm{D} u=\mathrm{D}^{2} x u-2 \mathrm{D} u+m \mathrm{D} x u-u u \\
& =(\mathrm{D}+m)(\mathrm{D} x u-2 u)+m u \\
x y= & x \mathrm{D} u+m x u=\mathrm{D} x u-u+m x u=(\mathrm{D}+m) x u-u .
\end{aligned}
$$

Let

$$
x\left\{\frac{d^{2} y}{d x^{2}}+(m+r) \frac{d y}{d x}+m r y\right\}+p\left(\frac{d y}{d x}+r y\right)=0 .
$$

If the above values be substituted in this, the result will have the factor $\mathrm{D}+m$ common to all the terms, and will reduce to

$$
x\left\{\frac{d^{2} u}{d x^{2}}+(m+r) \frac{d u}{d x}+m r u\right\}+(p-1)\left(\frac{d u}{d x}+r u\right)=0 .
$$

Consequently, if $y=(\mathbf{D}+m)^{p} u$, the proposed will reduce to

$$
\frac{d^{2} u}{d x^{2}}+(m+r) \frac{d u}{d x}+m r u=0 \text {. }
$$

Again, let $x\left(\frac{d^{2} y}{d x^{2}}+m \frac{d y}{d x}\right)+p \frac{d y}{d x}+n y=0$.

The same values substituted in this will give in like manner

$$
x\left(\frac{d^{2} u}{d x^{2}}+m \frac{d u}{d x}\right)+(p-1) \frac{d u}{d x}+n u=0 .
$$

Therefore $y=(\mathrm{D}+m)^{p} u$ will reduce it to 


$$
x\left(\frac{d^{2} u}{d x^{2}}+m \frac{d u}{d x}\right)+n u=0,
$$

which is more simple than the given equation.

'The equation

reduces to

$$
\frac{d^{2} y}{d x^{2}}+r x\left(\frac{d y}{d x}+m y\right)+r y=0
$$

where $y=(\mathbf{D}+m) u$.

$$
\frac{d^{2} u}{d x^{2}}+r x\left(\frac{d u}{d x}+m u\right)=0,
$$

With the same transformation we have

$$
\begin{gathered}
x^{2} \frac{d y}{d x}=(\mathrm{D}+m)\left(\mathrm{D} x^{2} u-4 x u\right)+2 m x u+2 u \\
x^{2} y=(\mathrm{D}+m) x^{2} u-2 x u ;
\end{gathered}
$$

and we shall find

$$
\frac{d^{2} y}{d x^{2}}+r x^{2}\left(\frac{d y}{d x}+m y\right)+2 r x y=0
$$

to transform immediately into

$$
\frac{d^{2} u}{d x^{2}}+r \cdot x^{2}\left(\frac{d u}{d x}+m u\right)=0 .
$$

Or to take a more general example,

$$
\frac{d^{2} y}{d x^{2}}+\left(r x^{2}+n x+k\right)\left(\frac{d y}{d x}+n y\right)+(2 r x+n) y=0
$$

will transform to

$$
\frac{d^{2} u}{d x^{2}}+\left(r x^{2}+n x+k\right)\left(\frac{d u}{d x}+m u\right)=0 .
$$

In the two last examples, where $y=(\mathrm{D}+m) u$, the transformed are more simple than the given equations.

'To give one more example of this sort of transformation, let

$$
\frac{d^{3} y}{d x^{3}}+m x^{2} \frac{d^{2} y}{d x^{2}}-2 m y=0 .
$$

Here we shall make $y=\mathrm{D} u+m x^{2} u$. Then

$$
\begin{aligned}
\frac{d^{3} y}{d x^{3}} & =\mathrm{D}^{4} u+m \mathrm{D}^{3} x^{2} u=\mathrm{D}^{4} u+m x^{2} \mathrm{D}^{3} u+6 m x \mathrm{D}^{2} u+6 m \mathrm{D} u \\
& =\left(\mathrm{D}+m x^{2}\right) \mathrm{D}^{3} u+6 m x \mathrm{D}^{2} u+6 m \mathrm{D} u=\left(\mathrm{D}+m x^{2}\right) \mathrm{D}^{3} u \\
& +6 m \mathrm{D}^{2} x u-6 m \mathrm{D} u .
\end{aligned}
$$

Also

$$
\begin{gathered}
x^{2} \frac{d^{2} y}{d x^{2}}=x^{2} \mathrm{D}^{3} u+m x^{2} \mathrm{D}^{2} x^{2} u=\mathrm{D}^{3} x^{2} u-6 \mathrm{D}^{2} x u+6 \mathrm{D} u \\
+m x^{2} \mathrm{D}^{2} x^{2} u=\left(\mathrm{D}+m x^{2}\right) \mathrm{D}^{2} x^{2} u-6 \mathrm{D}^{2} x u+6 \mathrm{D} u
\end{gathered}
$$


Substituting these values, the proposed equation becomes

$$
\left(\mathrm{D}+m x^{2}\right)\left(\mathrm{D}^{3} u+m \mathrm{D}^{2} x^{2} u-2 m u\right)=0 \text {; }
$$

or

$$
\mathrm{D}^{3} u+m \mathrm{D}^{2} x^{2} u-2 m u=0 \text {; }
$$

or

$$
\frac{d^{3} u}{d x^{3}}+m x^{2} \frac{d^{2} u}{d x^{2}}+4 m x \frac{d u}{d x}=0 \text {. }
$$

Make $\frac{d u}{d x}=z$, and the preceding becomes

$$
\frac{d^{2} z}{d x^{2}}+m x^{2} \frac{d z}{d x}+4 m x z=0
$$

which is only of the second order. The integration of it will give $z$. Then $y=\left(\mathrm{D}+m x^{2}\right) \mathrm{D}^{-1} z$.

Positive powers only of $\mathrm{D}$ have been used in these transformations; but it is obvious that we may employ negative ones also, and may make such assumptions as $y=\mathrm{D}^{-1} u+m u$, \&c. We may also use higher power's of $\mathrm{D}$, and we may express $y$ by more terms than two or three. And it is plain that we may apply a similar method to the transformation and integration of equations in partial differentials, and also to equations in finite differences. I shall close this paper with some examples of a more simple transformation.

Let

$$
\left(1-x^{3}\right) \frac{d^{3} y}{d x^{3}}-p(p-1)(p-2) y=0 .
$$

Make

$$
y=\mathrm{D}^{p-3} u \text {. }
$$

Then

$$
\mathrm{D}^{p} u-x^{3} \mathrm{D}^{p} u-p(p-1)(p-2) \mathrm{D}^{p-3} u=0,
$$

which by $(a$. $)$ reduces to

$$
\mathrm{D}^{p} u-\mathrm{D}^{p} x^{3} u+3 p \mathrm{D}^{p-1} x^{2} u-3 p(p-1) \mathrm{D}^{p-2} x u=0 ;
$$

or

$$
\mathrm{D}^{2} u-\mathrm{D}^{2} x^{3} u+3 p \mathrm{D} x^{2} u-3 p(p-1) x u=\mathrm{D}^{-p+2} 0=\mathrm{X}
$$

suppose, which is equivalent to

$$
\left(1-x^{3}\right) \frac{d^{2} u}{d x^{2}}+(3 p-6) x^{2} \frac{d u}{d x}-3(p-1)(p-2) x u=\mathrm{X},
$$

which is only of the second order.

Let

$$
\left(1-x^{3}\right) \frac{d^{3} y}{d x^{3}}+p(p+1)(p+2) y=0
$$

Make

$$
y=D^{-p-3} u \text {. }
$$

Then $\quad \mathrm{D}^{-p} u-x^{3} \mathrm{D}^{-p} u+p(p+1)(p+2) \mathrm{D}^{-p-3} u=0$

by (b.) will reduce to

$$
\left.\mathrm{D}^{-p} u-\mathrm{D}\right)^{-p} x^{3} u-3 p \mathrm{D}^{-p-1} x^{2} u-5 p(p+1) \mathrm{D}^{-p-2} x u=0 \text {; }
$$

or $\quad \mathrm{D}^{2} u-\mathrm{D}^{2} x^{3} u-3 p \mathrm{D} x^{2} u-3 p(p+1) x u=0$; 
or

$$
(1-x 3) \frac{d^{2} u}{d x^{2}}-(3 p+6) x^{2} \frac{d u}{d x}-3(p+1)(p+2) x u=0
$$

an equation of the second order only.

If

$$
\left(1+x^{3}\right) \frac{d^{3} y}{d x^{3}}+p(p-1)(p-2) y=0,
$$

$y=\mathrm{D}^{p-3} u$ will transform it to

$$
\left(1+x^{3}\right) \frac{d^{2} u}{d x^{2}}-(3 p-6) x^{2} \frac{d u}{d x}+3(p-1)(p-2) x u=\mathrm{D}^{-p+2} 0=\mathrm{X} .
$$

And if $\quad\left(1+x^{3}\right) \frac{d^{3} y}{d x^{3}}-p(p+1)(p+2) y=0$,

$y=\mathrm{D}^{-p-3} u$ will transform it to

$$
\left(1+x^{3}\right) \frac{d^{2} u}{d x^{2}}+(3 p+6) x^{2} \frac{d u}{d x}+3(p+1)(p+2) x u=0 .
$$

Gunthwaite Hall, October 20, 1846,

Near Barnsley, Yorkshire.

LXX. On the Electricity of Gun-Cotton. By Mr. J. E. Bowman, Demonstrator of Chemistry, King's College.

\section{To Richard Phillips, Esq.}

Dear Sir,

SINCE every one appears to be more or less engaged in the manufacture of the so-called "gun-cotton," it may be interesting to some of your readers to know that it is capable of being applied to a purpose different from any hitherto described, viz. that of insulating an electrically charged body. I was yesterday engaged in unravelling some cotton which had matted together while in the acid, when I was struck with the tenacity with which it adhered to my fingers; and on lightly holding a small flock of it, and approaching a finger of the other hand, or any foreign body, found that it was strongly attracted towards it, thus differing essentially from the unprepared cotton. I then subjected a little sewing cotton to the action of the acid, with the view of obtaining it in a more convenient form for testing its insulating power, and suspended from a brass rod two equally charged copper balls, one with white silk and the other with the prepared cotton. By examining the two balls at short intervals of time by means of a delicate gold-leaf electrometer, I found that the one suspended by the cotton retained its charge considerably longer 